DISKUSSIONSFORUM

\section{Innovationspolitische Aspekte der geplanten Einführung eines elektronischen Maut-Systems in Deutschland*}

\author{
von Günter Halbritter, Torsten Fleischer \\ und Christel Kupsch, ITAS
}

\begin{abstract}
Die vornehmlich als industriepolitische Panne angesehene, bisher an technischen und organisatorischen Problemen gescheiterte Einführung des technisch anspruchsvollen elektronischen Mautsystems Toll Collect in Deutschland offenbart auch innovationspolitische Defizite, die in Deutschland vorliegende politische Rahmenbedingungen für die im Augenblick intensiv diskutierten Innovationen betreffen. Nachfolgend werden innovationspolitische Aspekte der geplanten Einführung eines elektronischen Maut-Systems in Deutschland auf der Grundlage von Erfahrungen aus mehreren vom Bundesministerium für Bildung und Forschung (BMBF) geförderten Forschungsprojekten, die am Institut für Technikfolgenabschätzung und Systemanalyse (ITAS) des Forschungszentrums Karlsruhe durchgeführt werden und wurden, und die den Vergleich von Innovationsstrategien im Bereich der Verkehrstelematik in verschiedenen Ländern zum Untersuchungsgegenstand haben, kommentiert.
\end{abstract}

Vergleichende Untersuchungen von Innovationsstrategien im Bereich der Verkehrstelematik (VT) in verschiedenen Ländern, die vom ITAS durchgeführt wurden bzw. zurzeit noch laufen, zeigen, dass infrastrukturbasierte Innovationen, d. h. solche. deren Realisierung mit dem Aufbau von Infrastrukturleistungen verbunden ist, nicht nur einer konzeptionell-strategischen, sondern auch einer organisatorischen Begleitung durch staatliche Institutionen bedürfen, wenn sie erfolgreich realisiert werden sollen (Halbritter u. a. 1999; Halbritter u. a. 2002). In Deutschland hingegen wird Innovationsmanagement vornehmlich als Aufgabe der Industrie angesehen. Komplexe Aufgaben wurden auch im Falle von Toll Collect als Ganzes an die Industrie übergeben, in der Hoffnung, ihr wer- de die Realisierung schon gelingen. Die gesamte Einführungsstrategie, die Überprüfung vorgegebener bzw. festgelegter Schritte während der Einführungsphasen stehen damit nicht mehr im Einflussbereich der Politik. Die fehlenden Möglichkeiten des Eingreifens staatlicherseits bei der Koordinierung sind unter anderem als ein Grund für die Probleme bei der Einführung der satellitengestützten Lkw-Maut anzusehen.

Unstrittig ist der eigentliche Grund für die in den Medien als Maut-Desaster beschriebene Technikpanne eine Fehleinschätzung der Komplexität, die mit der Einführung neuer IuKTechniken im Rahmen integrativer Konzepte im großtechnischen Maßstab verbunden sind. Dabei ist im Rahmen von Toll Collect im Wesentlichen das organisatorische Problem der Systemintegration von Komponenten zu leisten, deren Basistechniken, wie z. B. GPS und GSM, weitgehend bekannt und erprobt sind. Das Scheitern dieser Systemintegration weist darauf hin, dass in jüngster Vergangenheit die Entwicklung technischer und organisatorischer Kompetenzen, die früher eine besondere Stärke der deutschen Industrie darstellten, hinter ökonomische Prioritätensetzungen zurückgetreten ist. So beklagen heute viele Ingenieure gerade der fortgeschrittenen Altersstufen, dass in den vergangenen Jahren der kreative, gestalterische Einfluss ingenieurwissenschaftlicher Kompetenz zurückgedrängt wurde. Diese Tendenz kennzeichnet besonders auch die Entwicklung im Bereich der Bahntechnik. Das Tragische an dieser Entwicklung ist, dass bei der Vielzahl der Pannen bei der Einführung neuer Techniken auch die ökonomischen Erwartungen nicht in Erfüllung gingen.

Insgesamt lässt sich in jüngster Vergangenheit die widersprüchliche Entwicklung beobachten, dass einerseits neue technische Produkte, wie Digitalkameras und Mobiltelefone, sich großen Interesses sowohl seitens der breiten Öffentlichkeit als auch der Medien erfreuen. Dem steht jedoch nur ein sehr schwach ausgeprägtes Interesse an den eigentlichen technischen Konzepten gegenüber, die Grundlage für diese neuen Produkte und Dienstleistungen sind. Dies spiegelt sich auch wider in dem schweren Stand, den kompetenter Technikjournalismus in Deutschland besitzt. So war und ist auch die mediale Diskussion im Falle der LkW-Maut gar nicht von technischen Problemen geprägt, selten 
ist von ihnen überhaupt die Rede, vielmehr spielen vornehmlich Spezialfragen der Vertragsgestaltung eine viel bedeutendere Rolle. Auch vom Verkehrsministerium vergebene Beraterverträge zielen dem Vernehmen nach vornehmlich auf rechtliche und wirtschaftliche Fragestellungen und nicht auf die technische Umsetzung von Mautkonzepten, andernfalls hätten kritische technische Machbarkeitsaspekte zumindest als mögliches Umsetzungsproblem angesprochen werden müssen.

Dieser mangelnde Bezug von Entscheidungsträgern und Multiplikatoren zu den grundsätzlichen Funktionsweisen technischer Konzepte ist auch ein Grund für unrealistische Visionen zu deren Leistungsfähigkeit und notwendiger Umsetzungszeiträume. Toll Collect ist ein Beispiel für eine solche utopischen Visionen entspringende Überschätzung technischer Systeme. Diese Überschätzung geht sogar so weit, dass in Deutschland, wie das Beispiel von Toll Collect ebenfalls zeigt, verkehrspolitische Überlegungen technischen und industriepolitischen Konzepten untergeordnet werden. Dies kann in kaum einem anderen Land so deutlich beobachtet werden wie in Deutschland. So besteht seit vielen Jahren bei den großen Parteien Konsens darüber, dass der Güterverkehr angemessene Anteile an den Infrastrukturkosten zu tragen hat und dass streckenbezogene Straßenbenutzungsgebühren das geeignete Instrument hierzu seien. Aber anstatt diese Einschätzung schon vor Jahren mit verfügbaren und erprobten terrestrischen Techniken umzusetzen, wird ein „Hightech“-Konzept entwickelt, dass bezüglich seiner technischen Auslegung - Gebührenabbuchung auch bei hohen Geschwindigkeiten und gleichzeitigem Spurwechsel - für den LkW-Verkehr absolut überdimensioniert ist. Die Realisierung einer vernünftigen verkehrspolitischen Einsicht muss daher auf die Entwicklung eines überdimensionierten „Hightech“-Konzepts warten.

\section{Der Staat als Innovationsmanager?}

Ein wesentliches Ergebnis der oben genannten, vom Bundesministerium für Bildung und Forschung (BMBF) geförderten Forschungsprojekte des ITAS, die sich mit Innovationsstrategien im Bereich der Verkehrstelematik in verschiedenen Ländern befasst haben, war, dass infrastrukturbasierte Projekte, d. h. Vorhaben, deren Reali- sierung mit dem Aufbau von Infrastrukturleistungen verbunden ist, nicht nur einer konzeptionell-strategischen, sondern auch einer organisatorischen Begleitung durch staatliche Institutionen bedürfen, um erfolgreich zu sein. Diese Erkenntnis der Notwendigkeit staatlichen Engagements über vertragsrechtliche Regelungen hinaus findet nicht nur in den USA, sondern auch auf der Ebene der Europäischen Union und einer Reihe europäischer Staaten Beachtung, die verkehrstelematische Konzepte entsprechend verkehrspolitischen Strategien teilweise bereits erfolgreich umgesetzt haben. So etwa in Frankreich, Italien, den Niederlanden, dem Vereinigten Königreich, Schweden, Finnland, Tschechien und Österreich. Deutschland hingegen verzichtet bisher auf die Entwicklung verkehrsstrategischer Konzepte zum Einsatz der Verkehrstelematik und hofft auf die Wirkung autonomer Marktmechanismen, ohne dass vorher entsprechende Rahmenbedingungen für einen solchen Markt entwickelt wurden. Dies geschieht auf der Grundlage von Fehlinterpretationen von Konzepten, wie das der „Public Private Partnership (PPP)“.

Besonders überraschend ist die innovationsstrategische Praxis in den USA, dem Land, das zumeist als Vorbild für erfolgreiche Innovationsaktivitäten gilt. Im Vergleich zu Deutschland ist dort ein bemerkenswert hohes staatliches Engagement bei der Konzeption und Durchsetzung von Innovationsstrategien im Bereich der Verkehrstelematik, dort ITS - Intelligent Transportation Systems genannt, festzustellen. Die Entwicklung und der Einsatz der neuen Techniken wird dabei keineswegs der Industrie allein überlassen, vielmehr fördern staatliche Institutionen nicht nur die Einführung von ITS in einer systematischen und konsequenten Weise, sondern begleiten diese auch in der Einführungsphase (deployment) und üben einen gezielt lenkenden Einfluss im Hinblick auf die angestrebten Zielvorgaben aus. Man ist geneigt, angesichts des Fördervolumens für nationale ITS-Programme von einem gigantischen staatlichen Technikeinführungsprogramm zu sprechen.

Die Vielzahl staatlicher Aktivitäten in diesem Bereich erweckt den Anschein eines Planungsperfektionismus, bei dem staatlichen Institutionen vornehmlich die Vorgabe der strategischen Ausrichtung zukommt. Insbesondere vier 
Aspekte kennzeichnen die US-amerikanischen Aktivitäten: So werden von den für die Verkehrspolitik verantwortlichen staatlichen Institutionen nicht nur zukunftsorientierte Programme festgelegt, sondern diese Programme bestimmen auch gesetzliche Regelungen zur Einführung und Umsetzung neuer Techniken und Dienste. Noch konkreter bezüglich der Umsetzung der neuen Techniken und Dienste sind die Vorgaben der so genannten nationalen Architektur, die sich nicht nur auf die Schnittstellenabstimmung verschiedener technischer Einzelmodule beziehen, sondern grundsätzliche Aspekte und Anforderungen für die Einführung neuer Techniken und Dienste beschreiben. Schließlich ist das systematische Projektmanagement nicht nur bei der Entwicklung, sondern auch beim Einsatz der neuen Techniken und Dienste in konkreten Anwendungszusammenhängen zu erwähnen.

Um dieses systematische Projektmanagement leisten zu können, wurden in den USA auch institutionelle Voraussetzungen im administrativen Bereich und bei der wissenschaftlichen Begleitung der Programme geschaffen. So wurde in den USA nicht nur im US-Verkehrsministerium (US-DoT) mit dem „Joint Program Office“ eine ressortübergreifende Querschnittsorganisation für die verschiedene Verkehrsträger betreffenden ITS-spezifischen Fragen eingerichtet, sondern auch spezielle wissenschaftliche Institutionen, wie das Volpe Center mit Begleituntersuchungen beauftragt, das mit seinen vielfältigen Kompetenzen im Bereich der strategischen Innovationsplanung und der unmittelbaren wissenschaftlichen Politikberatung in diesem Bereich sehr hilfreich ist.

Auch in der EU wurde, wie bereits erwähnt, die Notwendigkeit einer konzeptionellen Gestaltung der Verkehrstelematik im Hinblick auf verkehrs- und umweltpolitische Anforderungen erkannt und auch umgesetzt. So wurde eine „EU Rahmenarchitektur“ der Verkehrstelematik entwickelt, die Strukturen und Funktionen der neuen Techniken und Dienste beschreibt. Sie soll als Grundlage für die Entwicklung von „nationalen Architekturen“ dienen. Ein Großteil der EU-Länder haben die entsprechenden Vorgaben der EU auch in Form von „,nationalen Plänen“ und „nationalen Architekturen" bereits umgesetzt bzw. sie sind dabei, diese umzusetzen. In Deutschland wurde bisher jedoch keine Notwendigkeit gesehen, von Seiten der Regierung strategische und organisatorische Vorgaben zu machen.

Als wir im Jahre 1999 die US-amerikanische Vorgehensweise vor Vertretern des BMBF und des damaligen BMV, heute BMVBW, vorstellten, war der lakonische Kommentar des Vertreters des Verkehrsministeriums zu unseren Ausführungen, eine nationale Architektur werde es in Deutschland nicht geben. Von Seiten der Politik sei alles getan, jetzt sei die Industrie am Zuge und der Markt werde die Einführung der neuen Techniken und Dienste regeln. Eine Einschätzung, die schon deshalb sehr erstaunlich ist, da die USA nicht gerade das Land ist, wo Marktprozesse eine unbedeutende Rolle spielen.

\section{Unterschiedliches Verständnis von „Public Private Partnership (PPP)“}

Die unterschiedliche Einschätzung der Notwendigkeit staatlichen Engagements bei der Realisierung von Innovationsstrategien wird auch aus der unterschiedlichen Bedeutung des Begriffs „Public Private Partnership (PPP)“ deutlich. Dieser Begriff erfreut sich in Deutschland einer Beliebtheit, die weit über diejenige anderer Anglizismen hinausgeht. Dabei ist jedoch bemerkenswert, dass in den USA ganz andere Vorstellungen über die grundsätzliche Bedeutung und die praktische Realisierung von PPP bestehen als in Deutschland. Dort werden, wie bereits erwähnt, PPP-Projekte im Bereich der Verkehrstelematik vornehmlich auf der Grundlage staatlicher Strategiekonzepte und Programme durchgeführt. Privaten Unternehmen werden im Rahmen der Realisierung dieser Programme klar umrissene Arbeitspakete zugewiesen. Umfangreiche staatlich koordinierte Evaluationsprogramme begleiten die praktische Umsetzung. Um diese konzeptionellen Arbeiten zu leisten, wurden sowohl in der einzelstaatlichen wie auch der Bundes-Administration entsprechende organisatorische Voraussetzungen geschaffen. Auch stehen, wie bereits erwähnt, kompetente Einrichtungen zur wissenschaftlichen Politikberatung für diese komplexen Innovationsvorhaben zur Verfügung. Für mit deutschen Verhältnissen Vertraute erstaunt auch immer wieder die Deutlichkeit mit der in den Evaluationsberichten die noch vorliegenden Defizite benannt werden. So wird in dem Evaluationsbericht zu einem vom US-Verkehrsministerium in vier US-Ballungs- 
räumen durchgeführten Projekt zur Einführung von Telematikdiensten klar ausgesprochen, dass bisher kein einziges erfolgreiches PPPProjekt realisiert werden konnte (,...there was no successful PPP-project“; US-DoT 2001).

In Deutschland hingegen wird Innovationsmanagement vornehmlich als Aufgabe der Industrie angesehen. Es wurden zwar eine Reihe von Innovationsbeiräten gegründet, diese haben jedoch bisher keine wirklich gestaltenden Aktivitäten entfaltet. Im administrativen Bereich auf der Ebene des Bundes werden bestimmte technische Entwicklungslinien im Rahmen von Programmen des BMBF gefördert. Diese werden aber häufig - zumindest in der Frühphase unter einseitig industriepolitischen Aspekten ohne Einbeziehung der jeweils verantwortlichen Fachressorts und damit auch ohne frühzeitige Analyse der praktischen Umsetzungsmöglichkeiten vorangetrieben. Dies kann, wie das Beispiel des Magnetschwebebahnsystems Transrapid zeigt, zu erheblichen Problemen führen. Technisch durchaus interessante und viel versprechende Projekte scheitern, weil der Analyse der Umsetzbarkeit und der staatlichen Begleitung der Umsetzung (deployment) nicht frühzeitig der notwendige Stellenwert gegeben wird. In den angelsächsischen Ländern sind die Förderung von Technikentwicklungen wie auch die Einführung neuer Techniken zumeist Gegenstand der Fachadministrationen, die dadurch gezwungen sind, Einführungsstrategien zu entwickeln und entsprechende organisatorische Strukturen aufzubauen, die sich an den spezifischen Bedingungen der Einführung der neuen Techniken zu orientieren haben.

\section{Ineffektiver Innovationsdiskurs}

Das Beispiel des Mautsystems Toll Collect zeigt somit auch die erheblichen Auswirkungen, die Defizite im administrativen Bereich haben können. Komplexe Aufgaben wurden auch in diesem Fall als Ganzes an die Industrie übergeben, in der Hoffnung, ihr werde die Realisierung schon gelingen. Die gesamte Einführungsstrategie, die Überprüfung vorgegebener bzw. festgelegter Einführungsphasen stehen damit nicht mehr im Einflussbereich der Politik. Da weder nationale Programme noch entsprechende Gesetze für die Einführung der neuen Techniken als notwendig erachtet werden, sind diese auch nicht Gegenstand parlamentarischer Beratungen. Ohnehin ist die parlamentarische Arbeit in Deutschland nicht durch innovationspolitische Diskurse oder Initiativen gekennzeichnet, sieht man von wenigen Ausnahmen, wie dem Gentechnikgesetz, einmal ab. Das Parlament besitzt zwar kompetente Beratungseinrichtungen zur Problematik der Einführung von neuen Technologien, diese werden aber zumeist zur Beratung über langfristige Entwicklungen und weniger zur unmittelbaren Technikeinführung herangezogen. An dieser Situation ändert auch die vor kurzem initiierte Innovationsdebatte nichts, die bisher in keinem Fall konkret geworden ist oder Visionen derjenigen Innovationen entwickelte, die für die Zukunft von Bedeutung sein sollten, z. B. um die oft geforderte „nachhaltige Entwicklung“ zu befördern.

Dieser neuerlich in Deutschland initiierte Innovationsdiskurs, wenn man den Äußerungen der vergangenen Monate diese anspruchsvolle Bezeichnung zuordnen kann, bleibt auch deshalb oberflächlich, da dem mit der Forderung nach Innovationsbereitschaft verbundenen Anspruch an die Politik, in Legislative und Exekutive, nur unzureichend entsprochen wurde. Innovationen sind Umsetzungen technischer und organisatorischer Neuerungen in die gesellschaftliche Praxis. Sie erfordern daher auch entsprechende politische Rahmenbedingungen, um verwirklicht zu werden. Insbesondere die neuen Informations- und Kommunikationstechniken (IuK-Techniken) können ihre Systemvorteile erst dann entfalten, wenn die entsprechenden Bedingungen vorliegen.

Als Fazit bleibt zu betonen, dass es zu einfach wäre, die Panne bei Toll Collect allein der Industrie anzulasten, ebenso große Versäumnisse sind auch den politisch Verantwortlichen zu zuschreiben. Hier wird zwar gerne von Innovationen gesprochen, aber weder im Ministerialbereich noch auch im Parlament wurden Strukturen aufgebaut, um den mühsamen Prozess Innovationsmanagement als Umsetzungen technischer Neuerungen in der Gesellschaft - zu begleiten, so wie es in anderen Staaten geschieht. Es besteht die Gefahr, dass die am Beispiel Toll Collect deutlich gewordene mangelnde Bereitschaft, technischen Problemen eine entsprechende Bedeutung zuzumessen, zusammen mit dem mangelnden Interesse in der Gesellschaft, technische Probleme bezüglich ihrer 
Komplexität zur Kenntnis zu nehmen, auch andere Bereiche, wie etwa die Energieversorgung, treffen kann. Dies würde erhebliche Auswirkungen auf unsere technisch-zivilisatorisch geprägte Gesellschaftsstruktur haben. In diesem Sinne kann das Debakel von Toll Collect sogar einen positiven Besinnungsprozess auslösen über die Rolle, die der Technikentwicklung in der Gesellschaft zukommen sollte und wie diese zu gestalten und zu begleiten wäre.

* Überarbeitete Version des zuerst in der Zeitschrift Internationales Verkehrswesen, Band 56, Heft 9/2004, S. 363-366 veröffentlichten Beitrags.

\section{Literatur}

Halbritter, G.; Bräutigam, K.-R.; Fleischer, T.; Klein-Vielhauer, S.; Kupsch, Chr.; Paschen, H., 1999: Umweltverträgliche Verkehrskonzepte: Entwicklung und Analyse von Optionen zur Entlastung des Verkehrsnetzes und zur Verlagerung von Straßenverkehr auf umweltfreundlichere Verkehrsträger. Berlin u. a.: Erich Schmidt Verlag (Beiträge zur Umweltgestaltung A 143)

Halbritter, G.; Bräutigam, K.-R.; Fleischer, T.; Fulda, E.; Georgiewa, D.; Klein-Vielhauer, S.; Kupsch, Chr., 2002: Verkehr in Ballungsräumen: Beiträge von Verkehrstelematiktechniken und -diensten für einen effizienteren und umweltverträglicheren Verkehr. Berlin u. a.: Erich Schmidt Verlag (Beiträge zur Umweltgestaltung A 149)

US-DoT, 2001: Deploying and Operating Integrated Intelligent Transportation Systems. US-DoT Pub. No. 13599

\section{Kontakt}

Prof. Dr. Günter Halbritter

Forschungszentrum Karlsruhe in der Helmholtz-

Gemeinschaft

Institut für Technikfolgenabschätzung und Systemanalyse (ITAS)

Postfach 36 40, 76021 Karlsruhe

Tel.: +49 (0) 7247 / 82 - 4871

Fax: +49 (0) 7247 / 82 - 4806

E-Mail: halbritter@itas.fzk.de

Internet: http://www.itas.fzk.de

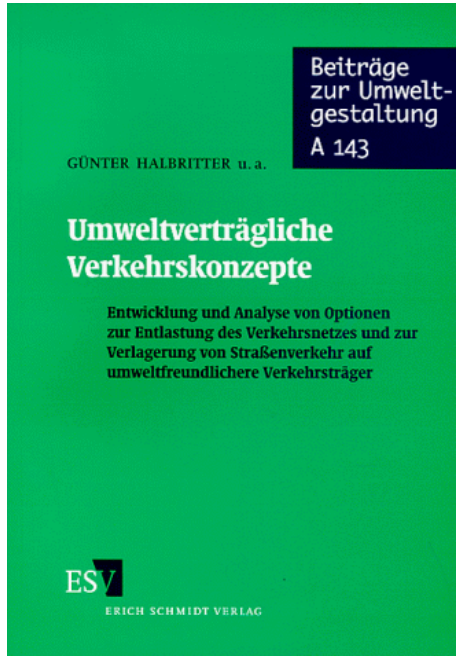

ISBN: 3-5030-4805 7; Preis: EUR 44,80

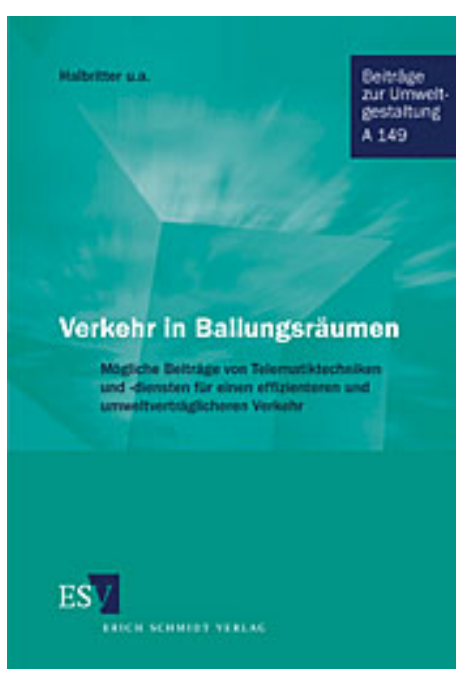

ISBN: 3-503-06686-1; Preis: EUR 29,80 